\title{
Assessment of compliance between a local "patients transportation protocol" and the latest European Consensus Recommendations for pediatric and neonatal retrieval in a COVID-19 context
}

\section{Cecile Dubois}

Paris Saclay University Hospitals

Valerie Ruetsch

Paris Saclay University Hospitals

Simon Barreault

Paris Saclay University Hospitals

Vincent Ollier

Paris Saclay University Hospitals

Gilles Jourdain ( $\nabla$ gilles.jourdain@aphp.fr)

Paris Saclay University Hospitals

\section{Short Report}

Keywords: COVID-19, SARS-CoV-2, Retrieval team, Personal protective equipment, Aerosol generating procedure

Posted Date: November 6th, 2020

DOl: https://doi.org/10.21203/rs.3.rs-103325/v1

License: (c) (i) This work is licensed under a Creative Commons Attribution 4.0 International License. Read Full License 


\section{Abstract}

As the COVID-19 pandemic evolves in Europe, safe transport of critically ill children challenged our retrieval team. In early July, recommendations for the transport of suspected or confirmed COVID-19 patients were published. Our study aimed to assess, retrospectively, if the recommendations were followed by our team. From the start of the current COVID-19 pandemic, our team developed transport procedures to protect health-care workers and to minimize nosocomial spread. The European Society of Pediatric Neonatal Intensive Care recommendations were made of a bunch of 17 elements. We extracted from our database all transports of suspected or confirmed COVID-19 from $1^{\text {st }}$ of March to 31th of May and compared for each transports the agreement with the 17 recommendations. Out of the 31 children that met the inclusion criteria, 29 were transported by our unit. Five recommendations were not applicable and therefore not discussed. Of the 12 recommendations remaining, 3 were not endorsed by our team: regarding the parental presence during retrieval, wearing a surgical mask for patient and the use of a dedicated pathway at arrival.

Conclusion: Most of the European recommendations were fully endorsed by our local retrieval team, but we have to improve our attitude in some aspects.

\section{Introduction}

The outbreak of SARS-CoV-2 infection emerged in December 2019 in Wuhan, Hubei Province, China. A series of adult patients with acute organ failure were first reported, followed by cases of SARS-CoV- 2 in children [1,2]. The medical staff had to manage the transportation of children with suspected or confirmed SARS-CoV-2 to reference centers. The ongoing COVID-19 outbreak resulting in high pressure on pediatric and neonatal retrieval teams. No official guidelines for pediatric retrieval were given in France so each team had to promote its "own recommendations", inspired by those published for adult transport [3] with added specificities, but always focusing on the main concern: safety for both patient and health-care workers. In early July, recommendations for the transport of suspected or confirmed COVID-19 patients were published by an expert panel from the European Society of Pediatric Neonatal Intensive Care (ESPNIC) [4]. This study aimed to retrospectively assess whether the European recommendations were closely adhered to our pediatric and neonatal retrieval team.

\section{Material And Methods}

Our pediatric retrieval team, located in the south-west of Paris (France), transports newborns and children under the age of 6 . The retrieval team is composed of 3 members: a driver, a nurse and a doctor. Since the beginning of the SARS-CoV-2 pandemic in France, we have issued a transport protocol for these patients as no other guidelines were yet to be published. Major concerns were related to the definition of the case, the choice of the ambulance (dedicated or not to COVID-19 patients' transfers), the use of personal protective equipment (PPE), full or partial depending on the proximity of the team member from the patient, airways management and aerosol-generating procedures (AGP), the circulation inside the 
receiving hospital and the communication with the receiving team and finally doffing and disinfection. Our local protocol was based on knowledge and preexisting protocols from adult teams and adult ICUs, to which we added a specific section regarding parental presence during retrieval. In early July, ESPNIC published the "European consensus recommendations for neonatal and pediatric retrievals of positive or suspected COVID-19 patients". These recommendations were elaborated with a modified Delphi method by a panel of experts and lead to 17 "strong" recommendations (Table 1). For this study, we retrospectively collected the data related to these transports from the $1^{\text {st }}$ of March to the end of May 2020 (end of the outbreak in France) using our medical database with the diagnosis "suspected or confirmed COVID-19" and assessed if our transports involving COVID-19 suspected or confirmed patients were compliant with these recommendations. For each transport, we rated each recommendation as 1 in case of agreement and 0 in case of total or partial disagreement, and N/A if not applicable for the transport. A previous study has shown that over $70 \%$ of agreement between experts results in a more favourable comprehensive systematic review-based process of selecting recommendations, and that agreement can be achieved within a much shorter time frame [5]. Therefore, we considered that we were in full agreement with ESPNIC's guidelines when the recommendations were followed more than $70 \%$ of the time.

The study was approved by the French National Commission for data protection (CNIL), under number 218975 as a standard reference procedure (MR004). Statistical analysis was performed using the global percentage of agreement for each recommendation.

\section{Results}

Over the period of interest, our retrieval team transported 31 patients with suspected or confirmed SARSCoV-2 infection ( 11 girls and 20 boys, sex ratio $=1,8$ ). The median age was respectively 205 days [36554]. Among these 31 patients: 10/31 (32,3\%) had noninvasive positive pressure ventilation (NIPPV), $2 / 31(6,5 \%)$ had high-flow nasal cannula (HFNC) and 2/31 (6,5\%) were intubated and mechanically ventilated.

Compliance between ESPNIC recommendations and our transports were reported in Table 1 and can be summarized as follows. Recommendations 7 and 8 were not applicable because we do not use videolaryngoscopy for intubation, and we did not elaborate on a specific COVID-19 related intubation protocol or checklist. In two cases $(6,5 \%)$ we only provided primary care, but children were not transported by our unit, therefore recommendations 6 to 16 were not applicable for those 2 . We decided not to discuss the recommendations involving less than 5 patients (numbers 6,13 and 14).

\section{Discussion}

This study reveals that our team complied with most of ESPNIC recommendations (over $70 \%$ of agreement in 9 out of 12 analyzed recommendations). 
For recommendation number 5 , only $7 / 29$ patients $(24 \%)$ wore a surgical mask to minimize aerosol spread. A recent study by Esposito et al [6] showed that it is not recommended for children under 2 years of age to wear any type of mask as these children with smaller airways can have trouble breathing if wearing one face mask. In our study, patients without a surgical mask (22/29) had a median age of 127 days [35; 427] and only 2 were over 2 years old. It is known that children have difficulty keeping their mask on and often try to remove it. Indeed, they end up touching their faces even more, and the use of masks can then lead to the nosocomial spread of the virus.

Regarding recommendation number 15 , the transport of infants and children without parents or relatives, regardless if symptomatic or not, sparked discussions within our team: in $69 \%$ of cases we had a full agreement. Parental accompaniment during inter-hospital transportation of critically ill children is common in our team practices. It has been described that taking one of the parents during transportation was feasible, beneficial and didn't increase staff stress or compromised the performance of medical interventions [7, 8]. It also potentially helped reduce anxiety for both patient and parent [9]. When parents were allowed to come in the ambulance, they were asked to wear full PPE as well. The retrieval team balanced the risk to create potential breaches in virus spread when transporting potentially infected parents, with the inconvenience to depart from our routine practice with increased major anxiety for the patient.

For recommendation 16 regarding the dedicated pathway to entering the receiving unit, we didn't comply with the ESPNIC's recommendation in 34,4\% ( $n=10)$ of cases. In fact, among the 10 transportations in question, we dropped patients in 7 different hospitals where the pathways for suspected COVID-19 weren't well signposted in hospitals, and most of the time at the beginning of the pandemic period (before the $1^{\text {st }}$ of April).

However, this study has some limitations. The interpretation of our results is limited by the small sample of the cohort and the retrospective nature of the study. We only explore our compliance with these recommendations, but it is not possible to measure whether if it was efficient to avoid the spread of the virus. We are now considering using the recommendations which met our full agreement in the context of other viral outbreaks such as bronchiolitis.

\section{Conclusion}

Most of the European recommendations were fully and easily endorsed by our team providing that guidelines had been elaborated for a realistic day-to-day use. Unorganized transport of infected children can lead to viral spread and parental presence during retrieval remains controversial during the outbreak of Covid-19. The use of surgical masks in the youngest patients is also very challenging and we still need to improve by using dedicated pathways towards the receiving units. These are the key improvement areas we need to focus on in anticipation of a second wave or reactivation of the outbreak of COVID-19.

\section{Declarations}




\section{Author declaration:}

- Ethical approval: The study was approved by the French National Commission for data protection (CNIL), under number 218975 as a standard reference procedure (MR004).

- Consent to participate: Information were provided to parents through a leaflet, seeking their nonopposition for study participation.

- Availability of data and materials: The datasets used and/or analysed during the current study are available from the corresponding author on reasonable request.

- Competing Interest: The authors declare that they have no conflict of interest.

- Funding: There is no funding source.

- Author contribution: CD, VR and GJ designed the study. CD collected the data. VR, GJ wrote the manuscript. VO and SB had input on the methods and text of the article. All authors contributed to and approved the final version of the text.

- Acknowledgements: N/A

\section{References}

1. Liu W, Zhang Q, Chen J, Xiang R, Song H, Shu S, Chen L, Liang L, Zhou J, You L, et al. Detection of Covid-19 in Children in Early January 2020 in Wuhan, China. N Engl J Med. 2020;382:1370-1371. doi: 10.1056/NEJMc2003717. Epub 2020 Mar 12.

2. Wu Z, McGoogan JM. Characteristics of and Important Lessons From the Coronavirus Disease 2019 (COVID-19) Outbreak in China: Summary of a Report of 72314 Cases From the Chinese Center for Disease Control and Prevention. JAMA 2020. Feb 24. doi: 10.1001/jama.2020.2648.

3. Yousuf B, Sujatha KS, Alfoudri H, Mansurov V. Transport of critically ill COVID-19 patients. Intensive Care Med. 2020 May 25:1-2. doi: 10.1007/s00134-020-06115-1

4. Terheggen U, Heiring C, Kjellberg M, Hegardt F, Kneyber M, Gente M, Roehr CC, Jourdain G, Tissieres P, Ramnarayan P, Breindahl M, van den Berg J. European consensus recommendations for neonatal and paediatric retrievals of positive or suspected COVID-19 patients. Pediatr Res. 2020 Jul 7. doi: 10.1038/s41390-020-1050-z. Online ahead of print.

5. Schoenberg NC, Barker AF, Bernardo J, Deterding RR, Ellner JJ, Hess DR, Maclntyre NR, Martinez FJ, Wilson KC. A Comparative Analysis of Pulmonary and Critical Care Medicine Guideline Development Methodologies. Am J Respir Crit Care Med. 2017 Sep 1;196(5):621-627 (2017). doi: 10.1164/rccm.201705-09260C

6. Esposito S, Principi N. To mask or not to mask children to overcome COVID-19. Eur J Pediatr. 2020 May 9:1-4. doi: 10.1007/s00431-020-03674-9.

7. Davies J, Tibby SM, Murdoch IA. Should parents accompany critically ill children during inter-hospital transport? Arch Dis Child. 2005 Dec;90(12):1270-3. Epub 2005 May 12.

doi:10.1136/adc.2005.074195 
8. Colville G, Orr F, Gracey D. "The worst journey of our lives": parents' experiences of a specialised paediatric retrieval service. Intensive Crit Care Nurs. 2003 Apr;19(2):103-8. doi: 10.1016/s09643397(03)00022-3

9. Woodward GA, Fleeger EW. Should parents accompany pediatric interfacility ground ambulance transports? The parent's perspective. Pediatric Emergency Care. 2000 Dec;10 16(6):383-90. doi: 10.1097/00006565-200012000-00001.

\section{Tables}

Title: ESPNIC's recommendations and agreement by the team

Caption: Second column lists all the recommendations, third shows our population and fourth the agreement by the retrieval team 
Number of patients

concerned in our

population (31)

our population

n

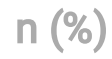

Determine status of the patient, COVID-19 positive or suspected, at referral of the patient Full PPE for transport staff involved in clinical patient care during the transport Reduced PPE for assisting transport staff not involved in patient care but within $<2 \mathrm{~m}$ of patient

Transport teams to train and simulate donning and doffing procedures of PPEs

Patient should whenever feasible wear a surgical mask to minimize aerosol spread Use cuffed endotracheal and tracheostomy tubes, including for newborn infants, to avoid aerosol spread by air leak

Use video-laryngoscopy whenever possible for intubation

Use strict protocol/checklist for the airway management/intubation

Use HEPA filters on the inspiratory limb of the ventilation circuit

Use HEPA filters on the expiratory limb, to avoid spreading contaminated air from the patient

No recommendation for or against the use of humidified air or heater/humidifier

Provide non-invasive ventilation with the use of a ventilator and a dual-limb system and filters as recommended above

Any incubator transport is to be handled like an open stretcher transport from a PPE point of view

Place newborn infant in suitably sized plastic bag to prevent heat loss and to reduce the need for incubator humidification Transport infants and children without parents or relatives, regardless if symptomatic or not. Assure secure and dedicated pathway for entering the receiving unit

Decontaminate any exposed transport equipment including equipment left within the transport vehicle.

\begin{tabular}{|l|l|}
\hline 31 & $26(84)$ \\
\hline 31 & $29(94)$ \\
\hline 31 & $25(81)$ \\
\hline 29 & $27(87)$ \\
\hline 2 & $7(24)$ \\
\hline 2 & $1(50)$ \\
\hline 5 & $\mathrm{~N} / \mathrm{A}$ \\
\hline 12 & $\mathrm{~N} / \mathrm{A}$ \\
\hline 12 & $12(100)$ \\
\hline 14 & $12(100)$ \\
\hline 12 & $14(100)$ \\
\hline 31 & $10(83)$ \\
\hline 3 & $31(100)$ \\
\hline 29 & $3(100)$ \\
\hline $3(0)$ \\
\hline $3(69)$ \\
\hline 3
\end{tabular}

